\title{
HtrA in Porphyromonas gingivalis can regulate growth and gingipain activity under stressful environmental conditions
}

\author{
F. Roy, $†$ E. Vanterpoolt‡ and H. M. Fletcher \\ Department of Biochemistry and Microbiology, School of Medicine, Loma Linda University, \\ Loma Linda, CA 92350, USA
}

Correspondence

H. M. Fletcher

hfletcher@llu.edu

Received 19 May 2006

Revised 17 July 2006

Accepted 21 July 2006

\begin{abstract}
In several micro-organisms, $\mathrm{HtrA}$, a serine periplasmic protease, is considered an important virulence factor that plays a regulatory role in oxidative and temperature stress. The authors have previously shown that the vimA gene product is an important virulence regulator in Porphyromonas gingivalis. Further, purified recombinant $\operatorname{VimA}$ physically interacted with the major gingipains and the $\mathrm{HtrA}$ from $P$. gingivalis. To further evaluate a role for $\mathrm{HtrA}$ in the pathogenicity of this organism, a $1.5 \mathrm{~kb}$ fragment containing the $h$ tr $A$ gene was PCR-amplified from the chromosomal DNA of $P$. gingivalis W83. This gene was insertionally inactivated using the ermF-ermAM antibiotic-resistance cassette and used to create an htrA-deficient mutant by allelic exchange. In one randomly chosen isogenic mutant designated $P$. gingivalis FLL203, there was increased sensitivity to hydrogen peroxide. Growth of this mutant at an elevated temperature was more inhibited compared to the wild-type. Further, in contrast to the wild-type, there was a significant decrease in Arg-gingipain activity after heat shock in FLL203. However, the gingipain activity in the mutant returned to normal levels after a further $30 \mathrm{~min}$ incubation at room temperature. Collectively, these data suggest that HtrA may play a similar role in oxidative and temperature stress in

$P$. gingivalis as observed in other organisms.
\end{abstract}

\section{INTRODUCTION}

Periodontitis is a destructive inflammatory disease of the supporting tissues of the teeth. An important aetiological agent implicated in chronic adult periodontitis is the Gramnegative, anaerobic, black-pigmented bacterium Porphryomonas gingivalis (reviewed by Genco et al., 1998, 1999; Griffen et al., 1999; Travis et al., 2000). This organism is also associated with other systemic diseases, including atherosclerosis (reviewed by Okuda \& Ebihara, 1998; Teng et al., 2002; Kinane \& Marshall, 2001). The major virulence factors of $P$. gingivalis, the gingipains, possess high levels of proteolytic activity and have been the focus of much attention (Genco et al., 1999; Imamura, 2003; Nakagawa et al., 2003). The major gingipains are extracellular and/or cell-associated. The Arg-specific gingipains, RgpA and RgpB, are encoded by the genes $r g p A$ and $r g p B$ respectively, whereas the Lys-specific protease (Kgp) is encoded by one gene, $k g p$ (Nakayama, 2003). There is a gap in our understanding of the regulation and processing of the gingipains. We have reported the posttranslational regulation of the gingipains in the vimAdefective mutant $P$. gingivalis FLL92 (Abaibou et al., 2001). The vimA gene is a part of the $b c p-r e c A$-vimA operon. Further,

†These authors contributed equally to this work.

łPresent address: Department of Biological Sciences, Oakwood College, Huntsville, AL, USA. protein-protein interaction studies using the purified rVimA showed that this protein interacts with the gingipains and the HtrA homologue in P. gingivalis (Vanterpool et al., 2006). The role, if any of $\mathrm{HtrA}$ in gingipain regulation and virulence in $P$. gingivalis is unclear.

Protein quality control, which is essential for bacterial survival, is regulated by chaperones and proteases $(\mathrm{Lu} \&$ McBride, 1994; Goulhen et al., 2003). HtrA (high temperature requirement $A$ ) is a periplasmic heat-shock serine protease that functions as a molecular chaperone at low temperatures and has proteolytic activity at elevated temperatures (Kim \& Kim, 2002). In several organisms, including bacteria, yeast, plant and humans, HtrA is considered an important factor involved in protein folding and maturation as well as in the degradation of misfolded proteins (Poquet et al., 2000). Inactivation of the htrA gene has been shown to affect the sensitivity of many organisms to thermal and oxidative stress (Pallen \& Wren, 1997; Lipinska et al., 1990). Here we report that HtrA physically interacts with the gingipains RgpA, RgpB and Kgp in P. gingivalis. HtrA is also involved in resistance to thermal and oxidative stress in $P$. gingivalis.

\section{METHODS}

Bacterial strains and growth conditions. Strains and plasmids used in this study are listed in Table 1. P. gingivalis strains were 
Table 1. Plasmids and bacterial strains

\begin{tabular}{|c|c|c|}
\hline Plasmid or strai & Phenotype/description & Source \\
\hline \multicolumn{3}{|l|}{ Plasmids } \\
\hline pCR2.1-TOPO & $\mathrm{Ap}^{\mathrm{r}} \mathrm{Km}^{\mathrm{r}}$ & Invitrogen \\
\hline pUC19 & $A p^{r}$ & Invitrogen \\
\hline pFLL200 & pCR 2.1-TOPO: htrA & This study \\
\hline pFLL201 & pUC19: htrA & This study \\
\hline pFLL202 & pUC19: htrA::ermF-ermAM & This study \\
\hline pVA2198 & $\mathrm{Sp}^{\mathrm{r}}$, ermF-ermAM & Fletcher et al. (1995) \\
\hline \multicolumn{3}{|l|}{$P$. gingivalis } \\
\hline W83 & Wild-type & This study \\
\hline FLL203 & htrA-defective mutant & This study \\
\hline \multicolumn{3}{|l|}{ E. coli } \\
\hline DH5 $\alpha$ & $\begin{array}{l}\mathrm{F}^{-} \phi 80 \mathrm{~d} l a c Z \Delta \mathrm{M} 15 \Delta(\text { lacZYA-argF }) \text { U169 recA1 endA1 } \\
\text { hsdR17 }\left(\mathrm{r}_{\mathrm{k}}^{-}, \mathrm{m}_{\mathrm{k}}^{-}\right) \text {phoA supE44 } \lambda^{-} \text {thi-1 gyrA96 relA1 }\end{array}$ & Invitrogen \\
\hline TOP10 & $\begin{array}{c}\mathrm{F}^{-} \text {mcrA } \Delta(\text { mrr-hsdRMS-mcrBC }) \phi 80 \text { lacZ } \Delta \mathrm{M} 15 \Delta \text { lacX74 recA1 } \\
\text { ara139 } \Delta(\text { ara-leu }) 7697 \text { galU galK rpsL }\left(\mathrm{Str}^{\mathrm{R}}\right) \text { endA1 nupG }\end{array}$ & Invitrogen \\
\hline
\end{tabular}

grown in brain heart infusion (BHI) broth (Difco) supplemented with haemin $\left(5 \mu \mathrm{g} \mathrm{ml}^{-1}\right)$, vitamin $\mathrm{K}\left(0.5 \mu \mathrm{g} \mathrm{ml}^{-1}\right)$ and cysteine $(0 \cdot 1 \%)$. Experiments with hydrogen peroxide were performed in BHI without cysteine. Escherichia coli strains were grown in LuriaBertani broth. Unless otherwise stated, all cultures were incubated at $37^{\circ} \mathrm{C}$. P. gingivalis strains were maintained in an anaerobic chamber (Coy Manufacturing) in $10 \% \mathrm{H}_{2} / 10 \% \mathrm{CO}_{2} / 80 \% \mathrm{~N}_{2}$. Growth rates for $P$. gingivalis and $E$. coli strains were determined by measuring $\mathrm{OD}_{600}$. Antibiotics were used at the following concentrations: clindamycin, $0.5 \mu \mathrm{g} \mathrm{ml}^{-1}$; erythromycin, $300 \mu \mathrm{g} \mathrm{ml}^{-1}$; carbenicillin, $100 \mu \mathrm{g} \mathrm{ml}^{-1}$.

DNA isolation and analysis. $P$. gingivalis chromosomal DNA was isolated as previously reported (Vanterpool et al., 2004). For plasmid DNA analysis, DNA extraction was performed by the alkaline lysis procedure as previously described (Johnson et al., 2004). For largescale preparation, plasmids were purified using the Qiagen plasmid maxi kit.

Generation of the $\boldsymbol{h t r A}$-defective mutant $\boldsymbol{P}$. gingivalis strain. A $1.5 \mathrm{~kb}$ fragment carrying the intact htrA ORF (gene number PG0535, http://www.oralgen.lanl.gov) was amplified by PCR using

Table 2. Primers

\begin{tabular}{|ll|}
\hline Primer & \multicolumn{1}{c|}{ Sequence $\left(\mathbf{5}^{\prime} \mathbf{- 3}^{\prime}\right)$} \\
\hline P1 $h$ trA forward & ATGAACAAAGTGTGGAAATAC \\
P2 $h$ trA reverse & TAGTTCTACTTCGAAGGTCCG \\
P3 pro-kgp forward & ATGAGGAAATTATTATTGCTGATCG \\
P4 pro-kgp reverse & TTGAAGAGCTGTTTATAAGCTGTTT \\
P5 erythromycin & TATTAGGCCTATAGCTTCCGCTATT \\
forward & \\
P6 erythromycin & AATAGGCCTTAGTAACGTGTAACTTT \\
reverse & \\
P7 sigA forward & ATGAGGCAACTTAAAATTTCC \\
F8 sigA reverse & TTAGCCGAGATAACCTTTCAG \\
\hline
\end{tabular}

primers $\mathrm{P} 1$ and $\mathrm{P} 2$ (Table 2). This fragment was cloned into the pCR2.1-TOPO plasmid vector (Invitrogen) and designated pFLL200. The $1.5 \mathrm{~kb}$ fragment was then isolated from pFLL200, digested with $B a m \mathrm{HI}$ and $\mathrm{XbaI}$ and ligated to pUC19 linearized with the same enzymes. The new recombinant plasmid was designated FLL201. Orientation was confirmed by restriction analysis. The ermF-ermAM cassette, which confers erythromycin/clindamycin resistance in $E$. coli and P. gingivalis, was PCR-amplified from pVA2198 using Pfu turbo (Stratagene) with primers P5 and P6 (Table 2). The amplified fragment was inserted into the MscI restriction site of the $h t r A$ gene. The resultant recombinant plasmid, pFLL202, was used as a donor in electroporation of $P$. gingivalis $\mathrm{W} 83$ as previously reported (Vanterpool et al., 2004).

Preparation of $\boldsymbol{P}$. gingivalis extracellular fractions and protease assays. One-litre cultures of $P$. gingivalis strains FLL203 and W83 were grown to stationary phase $\left(\mathrm{OD}_{600} 1 \cdot 5\right)$ from actively growing cells. Cells were harvested by centrifugation at $10000 \mathrm{~g}$ for $30 \mathrm{~min}$. The cell-free culture fluid was precipitated with cold $\left(-20{ }^{\circ} \mathrm{C}\right) 37.5 \%$ or $60 \%$ acetone, and the protein pellet was resus-

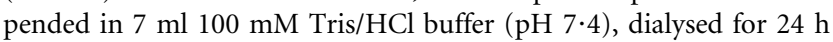
against the same buffer and then stored on ice at $0{ }^{\circ} \mathrm{C}$. The presence of Arg-X- and Lys-X-specific cysteine protease activities was determined with a microplate reader (Bio-Rad) as previously reported (Potempa et al., 1998).

Protein concentration determination. Protein concentration was calculated spectrophotometrically using an Eppendorf Biophotometer according to the manufacturer's instructions (Brinkman).

SDS-PAGE and immunoblot analysis. SDS-PAGE and transfer to nitrocellulose membranes were as described by Vanterpool et al. (2006). The blots were probed with antibodies against specific protease domains (Olango et al., 2003) or HagA (generously donated by Ann Progulske-Fox, University of Florida). The secondary antibody was immunoglobulin $\mathrm{G}$ (heavy plus light chains)-horseradish peroxidase conjugate (Zymed Laboratories). Immunoreactive proteins were detected by using the Western Lighting Chemiluminescence Reagent Plus kit (Perkin-Elmer Life Sciences).

Analysis of $\boldsymbol{P}$. gingivalis htrA-defective mutant genes by RTPCR. Total RNA was extracted from $P$. gingivalis strains grown to 
early stationary phase $\left(\mathrm{OD}_{600} 1 \cdot 2\right)$ using the RiboPure kit (Ambion). The primers used for RT-PCR analysis were specific for the kgp (primers $\mathrm{P} 3$ and $\mathrm{P} 4), h t r A(\mathrm{P} 1$ and $\mathrm{P} 2)$ and sigA genes (P7 and P8) (Table 2). The RT-PCR reaction $(50 \mu \mathrm{l})$ contained $1 \mu \mathrm{g}$ template RNA in the Superscript One-step RT-PCR reaction mix (Invitrogen). Negative controls were RT-PCR in the absence of reverse transcriptase.

Cell fraction preparation. One-litre cultures of $P$. gingivalis strains FLL203 and W83 were grown to stationary phase $\left(\mathrm{OD}_{600} 1 \cdot 5\right)$ from actively growing cells. Preparations of whole-cell culture, cell-free medium, cell suspension, vesicles and vesicle-free medium were made as previously reported (Olango et al., 2003). The whole-cell culture (WC) fraction is a sample of the culture after the bacterium has been grown to a specific growth phase. This sample has the bacterial cells suspended in the growth medium, and the enzyme activity includes the gingipains that are attached to the bacterial cell surface plus those secreted into the culture medium. After centrifugation, the cell pellet was resuspended, and the enzyme activity in this sample (the cell suspension fraction, CS) represents the gingipains that are attached to the bacterial cell surface. The enzyme activity in the supernatant (the cell-free medium fraction, CF) includes the gingipains that are secreted into the culture medium. Secreted gingipains can either be associated with vesicles or soluble in the culture medium; thus, ultracentrifugation of the cell-free fractions will yield a vesicle pellet (V) and a supernatant of soluble gingipains (VF).

Effect of heat shock on gingipain activity. Cultures of $P$. gingivalis strains FLL203 and W83 were grown for $16 \mathrm{~h}$ to an $\mathrm{OD}_{600}$ of $1 \cdot 5 ; 2 \mathrm{ml}$ samples from these cultures were incubated for $10 \mathrm{~min}$ at $55^{\circ} \mathrm{C}$ and proteolytic activities were analysed immediately and 30 min after heat shock treatment.

Growth at elevated temperatures and under oxidative stress conditions. Cultures $(100 \mathrm{ml})$ of $P$. gingivalis strains FLL203 and W83 were grown at $37^{\circ} \mathrm{C}$ for $16 \mathrm{~h}$ to an $\mathrm{OD}_{600}$ of $1 \cdot 5$. For growth at elevated temperatures, the cultures were then incubated at $42{ }^{\circ} \mathrm{C}$ under anaerobic conditions for $28 \mathrm{~h}$. Growth was determined at intervals of $0,4,8,24$ and $28 \mathrm{~h}$ as described above. For growth under oxidative stress conditions, cultures were grown in BHI without cysteine in the presence of $0.25 \mathrm{mM}$ hydrogen peroxide. Controls were grown (with cysteine) in the absence of hydrogen peroxide.

Cloning and expression of rHtrA. Oligonucleotide primers ( $\mathrm{P} 1$ and P2) specific for the ORF of the htrA gene were synthesized and used for PCR amplification. The amplified fragment carrying the htrA ORF was cloned into pTrcHis2-TOPO expression plasmid (Invitrogen) carrying coding for a C-terminal His-tag. The recombinant plasmid (designated pFLL137) was then transformed into competent E. coli TOP10F' cells (Invitrogen). Orientation of the htrA gene was determined by restriction endonuclease digestion. The nucleotide sequence of the insert was determined to rule out the occurrence of mutations. E. coli carrying the $h t r A$ ORF was grown in Luria-Bertani broth to exponential phase $\left(\mathrm{OD}_{600} 0 \cdot 7\right)$ in the presence of $50 \mu \mathrm{g}$ carbenicillin $\mathrm{ml}^{-1}$. IPTG was added at exponential phase and the cells were incubated at $37^{\circ} \mathrm{C}$ with shaking for an additional $6 \mathrm{~h}$. Cells were harvested by centrifugation and lysed by sonication. Cell membrane and debris were then harvested by centrifugation. The supernatant and pellet were analysed for the presence of the rHtrA protein. The presence of the poly-histidine tag was confirmed using the GelCode $6 \times$ His Protein Tag kit according to the manufacturer's instructions (Pierce).

Protein-protein interactions. The E. coli cells carrying the expressed rHtrA were lysed and the rHtrA was purified using $\mathrm{Ni}$ NTA magnetic beads. The purified rHtrA protein was then incubated for $30 \mathrm{~min}$ with magnetic beads coated with Ni-NTA. Beads were washed four times with wash/interaction buffers ( $50 \mathrm{mM} \mathrm{NaH} \mathrm{PO}_{4}, 300 \mathrm{mM} \mathrm{NaCl}, 50 \mathrm{mM}$ imidazole and $0.005 \%$ Tween 20). Cell lysates from $P$. gingivalis W83 grown to stationary phase were then incubated for $30 \mathrm{~min}$ with the with rHtrA-attached Ni-NTA magnetic beads. As a negative control, the lysates were incubated with the magnetic beads without the recombinant protein. After incubation with cell lysates, unbound proteins were eliminated by extensive washing in wash buffer. Proteins were eluted off the beads under denaturing conditions $(1 \times$ lithium dodecyl sulfate sample buffer, heated to $90{ }^{\circ} \mathrm{C}$ for $5 \mathrm{~min}$ ). Eluates were then analysed by SDS-PAGE and immunoblot analysis as described above.

\section{RESULTS}

\section{Inactivation of the htrA gene in $P$. gingivalis W83 by allelic-exchange mutagenesis}

Isogenic mutants of $P$. gingivalis W83 defective in the $h t r A$ gene were constructed by allelic-exchange mutagenesis. The recombinant plasmid pFLL202, which carries the ermFermAM cassette in the unique $M s c \mathrm{I}$ restriction site of the $h t r A$ gene, was used as a donor in electroporation of $P$. gingivalis W83. We predicted that two double-crossover events between the regions flanking the erm marker and the wild-type gene on the chromosome would result in replacement of a segment of the wild-type gene with a fragment conferring erythromycin resistance. Following electroporation and plating on selective medium (BHI containing $10 \mu \mathrm{g}$ erythromycin $\mathrm{ml}^{-1}$ ), we detected erythromycin-resistant colonies after a 7 day incubation period. To compare their phenotypic properties with those of wild-type strain W83, all mutants were plated on Brucella blood agar plates. Similar to the wild-type strain, all mutants displayed a black-pigmented, $\beta$-haemolytic phenotype.

\section{Confirmation of inactivation of htrA by PCR analysis}

Chromosomal DNA from six randomly chosen clindamycin/erythromycin-resistant colonies and the wild-type W83 strain was analysed by PCR to confirm the inactivation of the $h t r A$ gene. If the $h t r A$ gene was interrupted by the ermFermAM cassette, a $3.5 \mathrm{~kb}$ fragment was expected to be amplified using $h t r A$ primers $\mathrm{P} 1$ and $\mathrm{P} 2$. In addition, a $2 \cdot 1 \mathrm{~kb}$ fragment should be amplified from the mutant using the ermF-ermAM primers. The expected $3.5 \mathrm{~kb}$ (using $h t r A$ primers) and $2 \cdot 1 \mathrm{~kb}$ (using erythromycin primers) fragments were observed only in the erythromycin-resistant strains, not in the wild-type (Fig. 1a). To further confirm the absence of the htrA transcript in the erythromycin-resistant mutant, DNase-treated RNA from $P$. gingivalis FLL203 and the wild-type was subjected to RT-PCR. As shown in Fig. 1(b), no htrA transcript was detected from the mutant, in contrast to the wild-type W83. Using $k g p$-specific primers (Table 2) as a control, the expected $0 \cdot 8 \mathrm{~kb}$ fragment was amplified from all the $P$. gingivalis strains. No amplified fragment was observed from similar reactions in the absence of reverse transcriptase. These results indicated that the insertional inactivation of the chromosomal $h t r A$ gene with the $2 \cdot 1 \mathrm{~kb}$ ermF-ermAM antibiotic-resistance cassette was 
(a)

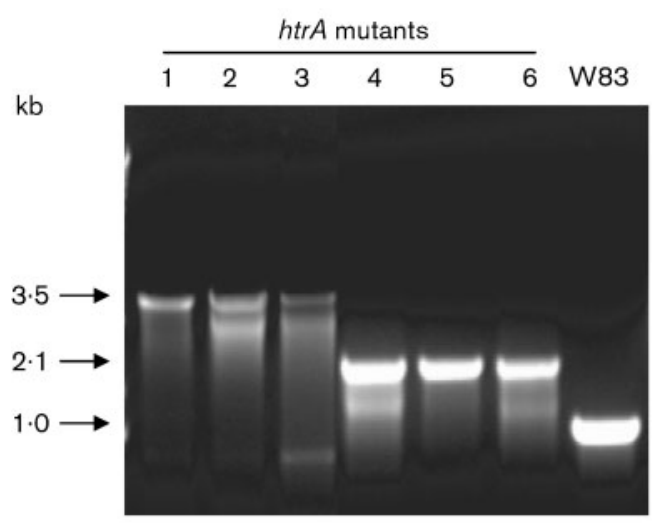

(b)

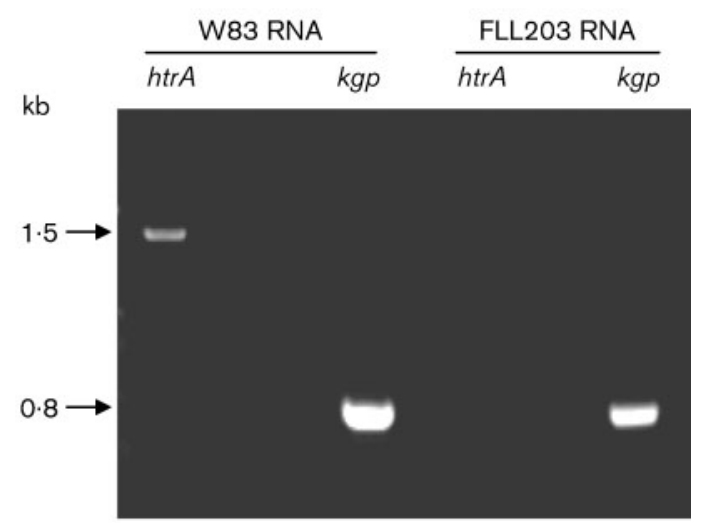

(c)

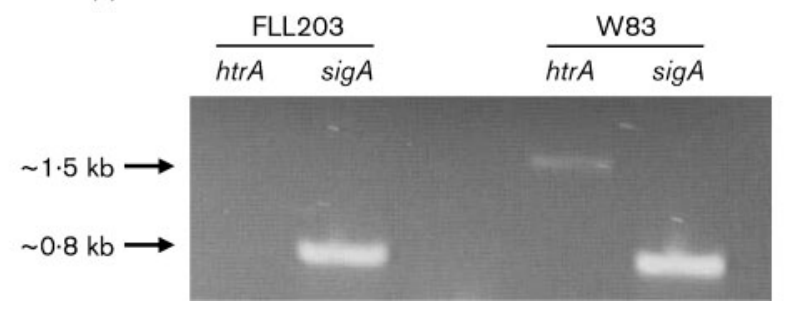

Fig. 1. Confirmation of inactivation by PCR and RT-PCR analysis. (a) Primers specific for the htrA gene (P1 and P2; Table 2) were used to amplify it from total cellular DNA from $P$. gingivalis: lane 1, P. gingivalis FLL203.1 (htrA : : ermF-ermAM); lane 2, P. gingivalis FLL203.2 (htrA : : ermF-ermAM); lane 3, P. gingivalis FLL203.3 (htrA : : ermF-ermAM) ; lane 7, P. gingivalis W83 (wild-type). Primers specific for the ermF-ermAM cassette (P5 and P6) were used to amplify the 2.1 cassette: lane $4, P$. gingivalis FLL203.1 (htrA::ermF-ermAM); lane 5, P. gingivalis FLL203.2 (htrA::ermF-ermAM); lane 6, P. gingivalis FLL203.3 ( $h$ trA : : ermF-ermAM). (b) Total RNA isolated from $P$. gingivalis W83 and the htrA-defective mutant in the stationary phase $\left(\mathrm{OD}_{600} 1 \cdot 4-1 \cdot 5\right)$ was subjected to RT-PCR using primers for $h$ tr $A$ and pro-kgp. No transcription of $h$ trA was detected in $P$. gingivalis FLL203 when reactions were performed in the absence of reverse transcriptase or in the negative controls. (c) DNase-treated RNA from $P$. gingivalis FLL203 and W83 grown to stationary phase was subjected to RT-PCR for the transcription of the sigA gene. For the analysis of $h t r A$, primers $\mathrm{P} 1$ and $\mathrm{P} 2$ were used. For the analysis of the sigA gene, primers P7 and P8 were used (Table 2).

successful. One mutant designated $P$. gingivalis FLL203 was randomly chosen for further study. The wild-type and the htrA-defective mutant FLL203 showed a similar generation time of $3 \mathrm{~h}$ (data not shown). To rule out any polar effect on the gene downstream of $h t r A$, DNase-treated RNA from FLL203 was further analysed by RT-PCR. Using specific primers (P7 and P8; Table 2) for the downstream sigA gene, a fragment of the predicted size was amplified from both the htrA-defective mutant and the wild-type (Fig. 1c).

\section{P. gingivalis FLL203 is more sensitive to oxidative stress than the wild-type}

P. gingivalis W83 and the htrA-defective mutant FLL203 were evaluated for oxidative stress resistance by their ability to grow in the presence of hydrogen peroxide. Compared to the parent strain, FLL203 demonstrated increased sensitivity to hydrogen peroxide at a final concentration of $0.25 \mathrm{mM}$ (Fig. 2a). The greatest sensitivity was observed during the stationary growth phase (Fig. 2b).

\section{Growth and Rgp activity at elevated temperatures are affected in $\boldsymbol{P}$. gingivalis FLL203}

P. gingivalis FLL203, the $h t r A$-defective mutant, grew at a similar rate to that of the wild-type at $37^{\circ} \mathrm{C}$ (data not shown). However, in contrast to the parent strain, the mutant grew more slowly at $42{ }^{\circ} \mathrm{C}$, and in addition did not grow over an $\mathrm{OD}_{600}$ of 0.9 (Fig. 3a). In cells grown at $42^{\circ} \mathrm{C}$ for 8 h or 28 h, Rgp activity was increased by approximately $20 \%$ and $10 \%$ respectively in the mutant compared to the wild-type strain (Fig. 3b). In contrast to the parent strain at a similar cell density, there was greater Rgp activity in the HtrA-defective mutant grown at $42{ }^{\circ} \mathrm{C}$ (Fig. 3c). The ability of $P$. gingivalis FLL203 to recover from heat shock was also evaluated. After a $10 \mathrm{~min}$ incubation at $55^{\circ} \mathrm{C}$, both the wildtype and the mutant strains were further incubated for $24 \mathrm{~h}$ at $37^{\circ} \mathrm{C}$. A slightly longer lag phase was observed in the $h t r A-$ defective mutant in comparison to the wild-type (data not shown). To determine if the gingipains are affected by brief 

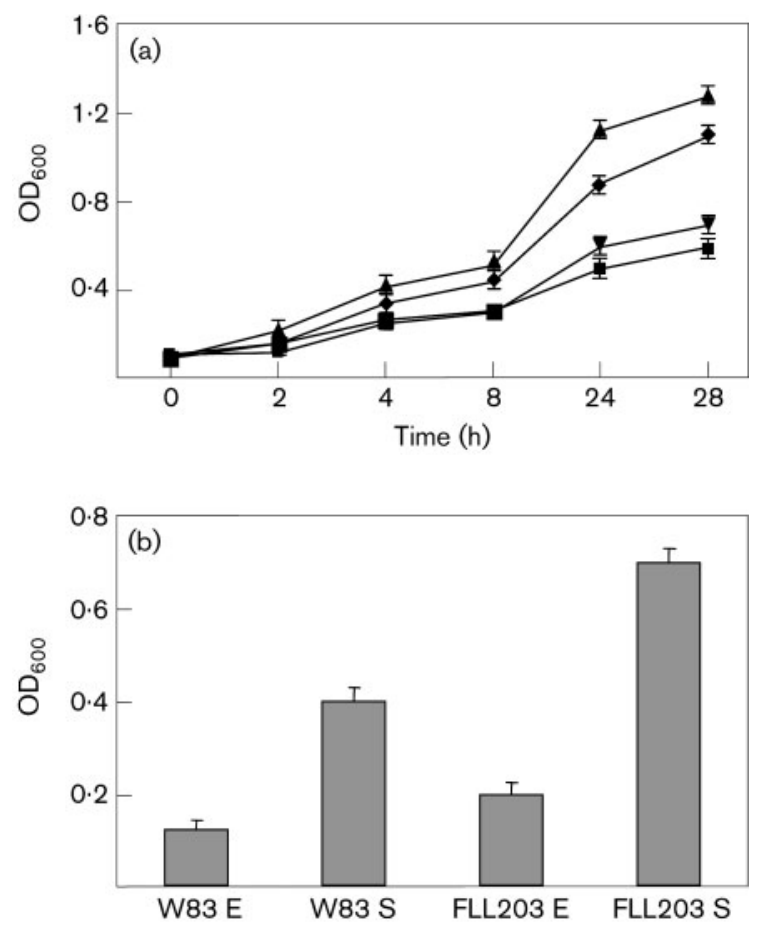

Fig. 2. The $h t r A$-defective mutant is more sensitive to hydrogen peroxide than the wild-type. Actively growing $P$. gingivalis W83 and FLL203 were incubated with $0.25 \mathrm{mM}$ hydrogen peroxide in the absence of cysteine. Sensitivities to hydrogen peroxide were determined by measuring $\mathrm{OD}_{600}$ over time. (a) $\boldsymbol{\Delta}$, FLL203 control; $\mathbf{\square}$, FLL203 treated; $\boldsymbol{\nabla}$, W3 control, $\boldsymbol{\nabla}$, W83 treated. (b) Difference in $\mathrm{OD}_{600}$ between treated and untreated cultures at exponential (E) and stationary (S) phase. The data in both panels are means $\pm S E$ of three independent experiments.

incubation at elevated temperatures in the $h \operatorname{tr} A$-defective mutant, $P$. gingivalis W83 and FLL203 were incubated at $55^{\circ} \mathrm{C}$ for $10 \mathrm{~min}$. At $37^{\circ} \mathrm{C}$, there was no significant difference in Rgp activity between the mutant and the wildtype (data not shown). However, immediately after heat treatment, Rgp activity in the wild-type was elevated by $7 \%$ in comparison to the untreated control. Interestingly, the Rgp activity in the htrA-defective mutant was decreased by $13 \%$ in comparison to the untreated control. Rgp activities determined $30 \mathrm{~min}$ after heat treatment were similar for the wild-type and FLL203 (data not shown).

\section{Gingipain profiles and distribution are altered under temperature stress}

The distribution of gingipains in the wild-type (Fig. $4 \mathrm{a}$ ) and the $h$ trA-defective mutant (Fig. $4 \mathrm{~b}$ ) was very similar under normal growth conditions. However, the distribution of the Rgps appeared to be slightly altered by incubations at $42^{\circ} \mathrm{C}$. There appeared to be approximately $30 \%$ less cellassociated Rgp activity in the $h t r A$-defective mutant (Fig. 4d) in comparison to the wild-type (Fig. 4c). Very little alteration in Kgp activity was observed (data not
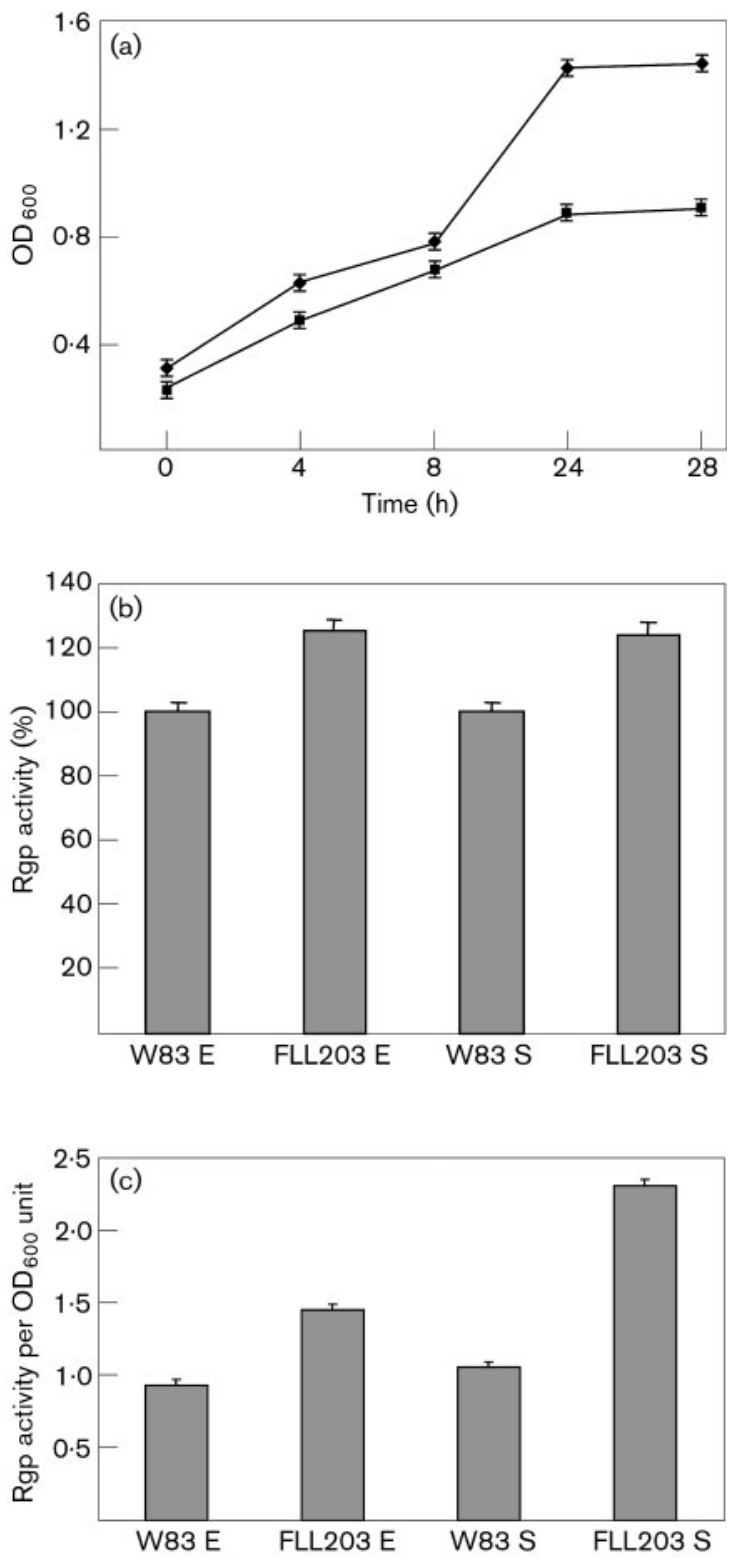

Fig. 3. Growth and Rgp activity are affected in $P$. gingivalis FLL203 at elevated temperatures. Cultures of $P$. gingivalis W83 and FLL203 were grown at $42{ }^{\circ} \mathrm{C}$ for $28 \mathrm{~h}$ under anaerobic conditions. (a) Growth at $42^{\circ} \mathrm{C}$ : $\mathbf{\square}$, FLL203; $>$, W83. (b) Rgp activity at exponential (E) and stationary (S) growth phase. (c) Rgp activity per $\mathrm{OD}_{600}$ unit. The error bars represent SE.

shown). Immunoblot analysis of extracellular fractions demonstrated the absence of RgpA and RgpB immunoreactive bands in the htrA-defective mutant (data not shown).

\section{DISCUSSION}

The inflammatory microenvironment of the periodontal pocket (Chapple, 1997; Katsuragi et al., 2003; Wolff et al., 

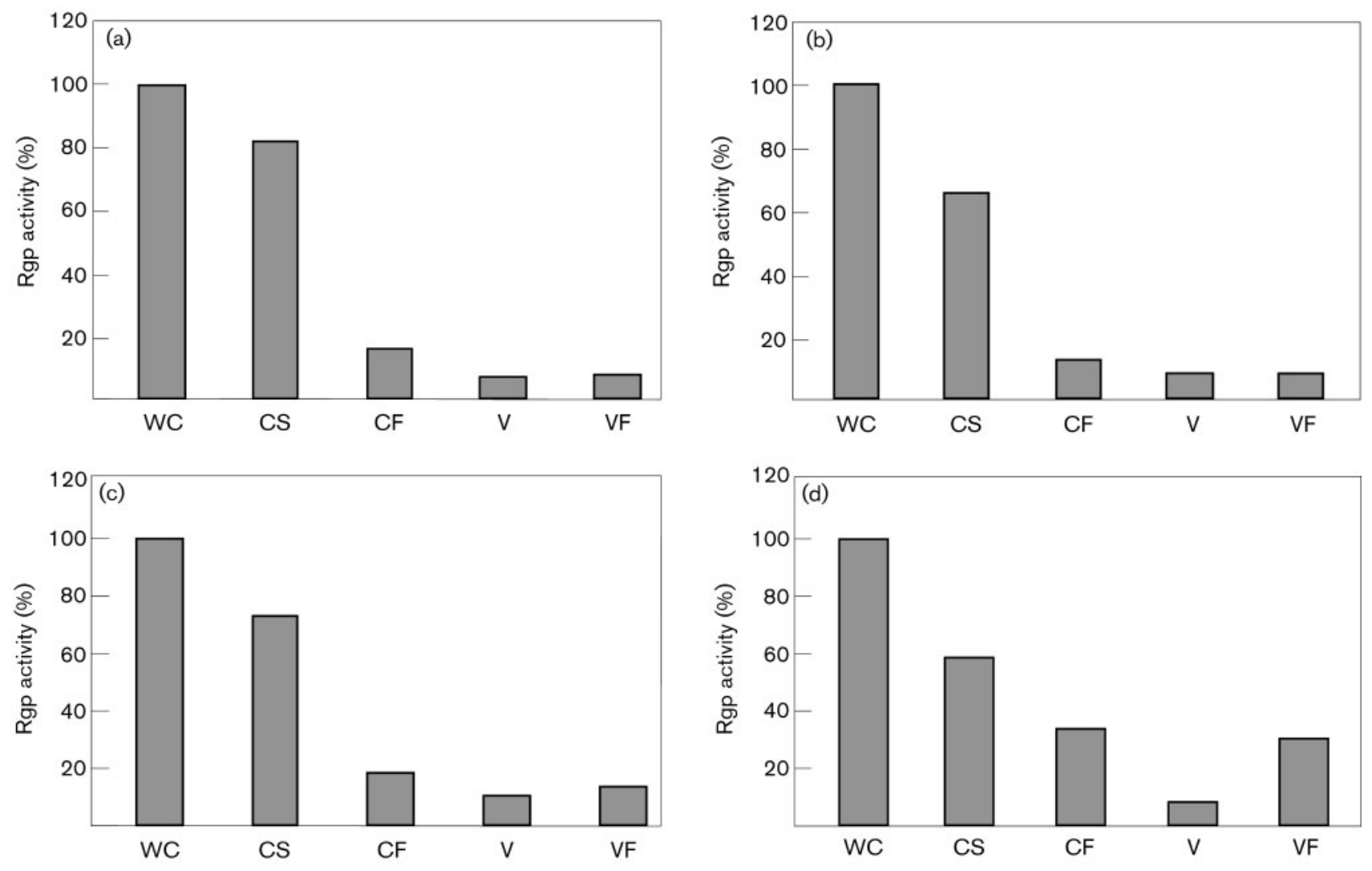

Fig. 4. Distribution of Rgp activity is altered in the $h t r A$-defective mutant. $P$. gingivalis FLL203 and W83 were grown at either $37^{\circ} \mathrm{C}$ or $42^{\circ} \mathrm{C}$. Activities against $N^{\alpha}$-benzoyl-DL-arginine- $p$-nitroanilide (BApNA) were tested in whole cell culture (WC), cell suspension (CS), cell free medium (CF), vesicles (V) and vesicle-free (VF) fractions of the $P$. gingivalis strains (see Methods for details). (a) W83 grown at $37^{\circ} \mathrm{C}$, (b) FLL203 grown at $37^{\circ} \mathrm{C}$, (c) W83 grown at $42{ }^{\circ} \mathrm{C}$, (d) FLL203 grown at $42{ }^{\circ} \mathrm{C}$.

1997) would suggest that factors that are important in oxidative and temperature stress would be critical for the survival of $P$. gingivalis. In many organisms, $\mathrm{HtrA}$ is known to play a regulatory role in response to environmental signals and is protective against oxidative and temperature stress (Cortes et al., 2002; Foucaud-Scheunemann \& Poquet, 2003; Lipinska et al., 1990; Lyon \& Caparon, 2004; Noone et al., 2000; Pallen \& Wren, 1997; Poquet et al., 2000). HtrA is also involved in the biogenesis of bacterial virulence factors. Thus its inactivation results in attenuated virulence (Cortes et al., 2002; az-Torres \& Russell, 2001; Lyon \& Caparon, 2004). In our study, we further evaluated the role of $\mathrm{HtrA}$ in $P$. gingivalis. We have demonstrated that the htrA-defective mutant is more sensitive to elevated temperature than the wild-type. In another study, L. Yuan \& A. Progulske-Fox (unpublished) demonstrated that the survival of the htrA-defective mutant is not significantly affected by a $50{ }^{\circ} \mathrm{C}$ heat shock. Collectively, these data may suggest that HtrA may play a role in long-term adaptation to prolonged elevated temperature, as demonstrated in this study. This could have implications for growth in the periodontal pocket, where there is usually increased temperature due to inflammation.
In addition to the altered growth of the $h t r A$-defective mutant at elevated temperature, there was an increase in gingipain activity in the mutant in comparison to the wildtype. The decreased cell density of the htrA-defective mutant at elevated temperature compared to the wild-type may indicate that the level of gingipain activity is significant. Our results indeed indicated that at a similar cell density, there was significantly more gingipain activity in the mutant than in the wild-type. Collectively, these results could suggest an involvement of HtrA in gingipain regulation at elevated temperatures. We are currently investigating the molecular changes that occur with the gingipains under prolonged growth at $42{ }^{\circ} \mathrm{C}$. Prolonged growth of $P$. gingivalis at temperatures above $37^{\circ} \mathrm{C}$ has previously been observed to result in a significant decrease in gingipain activity (Amano et al., 1994; Forng et al., 2000; Lynch \& Kuramitsu, 1999; Murakami et al., 2004a, b; Percival et al., 1999). Under these environmental conditions, it is likely that the decrease in proteolytic activity could be correlated with a downregulation in the expression of the gingipain genes (Percival et al., 1999). HtrA and other members of this family, including DegS and DegP, have a stress sensor function and are associated with the periplasmic side of the 
bacterial membrane (Day \& Hinds, 2002; Krojer et al., 2002; Pallen \& Wren, 1997). The stress sensor function is modulated via the PDZ domain and initiates a cascade of events that results in the induced expression of several genes critical to the stress response (Day \& Hinds, 2002; Krojer et al., 2002; Pallen \& Wren, 1997). A comparison of the $P$. gingivalis $\mathrm{HtrA}$ homologue with those of other known organisms reveals a PDZ domain and an N-terminal hydrophobic region, indicating membrane association (http://www.tigr.org) (van \& Hendriks, 2003; Pallen \& Wren, 1997). The mechanism for the regulatory role of HtrA in the expression of the gingipain genes in $P$. gingivalis is unknown. Other virulence genes in $P$. gingivalis are known to be downregulated at elevated temperature. However, it is unclear if a common mechanism is involved in their regulation. This is the subject of further investigation in our laboratory.

In this study, the gingipain activity in response to heat shock appeared to be different from the response of the organism grown at elevated temperature for an extended period. Decreased gingipain activity was observed in the htrAdefective mutant, in contrast to the wild-type, which showed an increase in activity. It is possible that during heat shock, denaturation of the gingipains could lead to decreased gingipain activity. In the wild-type, it is likely that the presence of the HtrA protein may stabilize the gingipains. $\mathrm{HtrA}$ is also known to have a chaperone function, and could play a role in the protection of the gingipains under these conditions, although we cannot rule out the possibility of other heat-shock proteins being involved. Preliminary results from our laboratory have demonstrated that in protein-protein interaction experiments with $\mathrm{rHtrA}$, this protein can interact with the gingipains as well as HagA (data not shown). These findings are currently being confirmed. In addition, HtrA can interact with the VimA protein, which can bind to the gingipains and regulate their activity (Vanterpool et al., 2006). Collectively, these data could further support a direct involvement of HtrA in the regulation of gingipain activity. It is likely that a complex of proteins stabilize the gingipains at elevated temperature, although HtrA is not essential for the maturation/activation of the gingipains under normal conditions. We cannot rule out the possibility that HtrA may regulate the expression of other heat-shock proteins that would be missing in the htrAdefective mutant. Several heat-shock proteins in $P$. gingivalis, including GroES, GroEL, DnaK and HtpG, have been documented (Yamazaki et al., 2004; Yoshida et al., 1999; Goulhen et al., 2003; Maeda et al., 1994).

In addition to having elevated temperatures, the periodontal pocket is an oxidative environment due to the presence of reactive oxygen species (Chapple, 1997; Katsuragi et al., 2003; Sculley \& Langley-Evans, 2002). Consistent with reports of other organisms (Ibrahim et al., 2004; Mutunga et al., 2004; Wonderling et al., 2004), our data also suggest that the htrA-defective mutant is more susceptible to hydrogen peroxide than the wild-type. This was also observed in a similar study by L. Yuan \& A. ProgulskeFox (unpublished). Given the multiple mechanisms of oxidative stress resistance in $P$. gingivalis, the relative significance of HtrA in this process is unclear. The virulence potential of the htrA-defective mutant was altered compared to that of the wild-type (L. Yuan \& A. Progulske-Fox, unpublished).

In conclusion, we can envision a scenario in $P$. gingivalis where the HtrA protein may be important for regulation of gingipain activity in the inflammatory microenvironment of the periodontal pocket. A specific mechanism for this interaction will be the subject of further study.

\section{ACKNOWLEDGEMENTS}

This work was supported by Loma Linda University School of Dentistry and by Public Health Service grants DE13664 and DE13664S1 from the National Institute of Dental and Craniofacial Research (to H.M.F.) and GM60507, a minority training grant from the National Institute of General Medicine. We would also like to thank Dr Jon Potempa for the gingipain antibodies and Ann Progulske-Fox for monoclonal HagA antibodies.

\section{REFERENCES}

Abaibou, H., Chen, Z., Olango, G. J., Liu, Y., Edwards, J. \& Fletcher, H. M. (2001). vimA gene downstream of $\operatorname{rec} A$ is involved in virulence modulation in Porphyromonas gingivalis W83. Infect Immun 69, 325-335.

Amano, A., Sharma, A., Sojar, H. T., Kuramitsu, H. K. \& Genco, R. J. (1994). Effects of temperature stress on expression of fimbriae and superoxide dismutase by Porphyromonas gingivalis. Infect Immun 62, 4682-4685.

az-Torres, M. L. \& Russell, R. R. (2001). HtrA protease and processing of extracellular proteins of Streptococcus mutans. FEMS Microbiol Lett 204, 23-28.

Chapple, I. L. (1997). Reactive oxygen species and antioxidants in inflammatory diseases. J Clin Periodontol 24, 287-296.

Cortes, G., de Astorza, B., Benedi, V. J. \& Alberti, S. (2002). Role of the htrA gene in Klebsiella pneumoniae virulence. Infect Immun $\mathbf{7 0}$, 4772-4776.

Day, C. L. \& Hinds, M. G. (2002). HtrA - a renaissance protein. Structure 10, 737-739.

Fletcher, H. M., Schenkein, H. A., Morgan, R. M., Bailey, K. A., Berry, C. R. \& Macrina, F. L. (1995). Virulence of a mutant of Porphyromonas gingivalis W83 that is defective in the prtH gene. Infect Immun 63, 1521-1528.

Forng, R. Y., Champagne, C., Simpson, W. \& Genco, C. A. (2000). Environmental cues and gene expression in Porphyromonas gingivalis and Actinobacillus actinomycetemcomitans. Oral Dis 6, 351-365.

Foucaud-Scheunemann, C. \& Poquet, I. (2003). HtrA is a key factor in the response to specific stress conditions in Lactococcus lactis. FEMS Microbiol Lett 224, 53-59.

Genco, C. A., Van Dyke, T. \& Amar, S. (1998). Animal models for Porphyromonas gingivalis-mediated periodontal disease. Trends Microbiol 6, 444-449.

Genco, C. A., Potempa, J., Mikolajczyk-Pawlinska, J. \& Travis, J. (1999). Role of gingipains $\mathrm{R}$ in the pathogenesis of Porphyromonas gingivalis-mediated periodontal disease. Clin Infect Dis 28, 456-465. 
Goulhen, F., Grenier, D. \& Mayrand, D. (2003). Oral microbial heatshock proteins and their potential contributions to infections. Crit Rev Oral Biol Med 14, 399-412.

Griffen, A. L., Lyons, S. R., Becker, M. R., Moeschberger, M. L. \& Leys, E. J. (1999). Porphyromonas gingivalis strain variability and periodontitis. J Clin Microbiol 37, 4028-4033.

Ibrahim, Y. M., Kerr, A. R., McCluskey, J. \& Mitchell, T. J. (2004). Control of virulence by the two-component system $\mathrm{CiaR} / \mathrm{H}$ is mediated via HtrA, a major virulence factor of Streptococcus pneumoniae. J Bacteriol 186, 5258-5266.

Imamura, T. (2003). The role of gingipains in the pathogenesis of periodontal disease. J Periodontol 74, 111-118.

Johnson, N. A., Liu, Y. \& Fletcher, H. M. (2004). Alkyl hydroperoxide peroxidase subunit $\mathrm{C}(a h p C)$ protects against organic peroxides but does not affect the virulence of Porphyromonas gingivalis W83. Oral Microbiol Immunol 19, 233-239.

Katsuragi, H., Ohtake, M., Kurasawa, I. \& Saito, K. (2003). Intracellular production and extracellular release of oxygen radicals by PMNs and oxidative stress on PMNs during phagocytosis of periodontopathic bacteria. Odontology 91, 13-18.

Kim, D. Y. \& Kim, K. K. (2002). Crystallization and preliminary X-ray studies of the protease domain of the heat-shock protein HtrA from Thermotoga maritima. Acta Crystallogr D Biol Crystallogr 58, 170-172.

Kinane, D. F. \& Marshall, G. J. (2001). Periodontal manifestations of systemic disease. Aust Dent J 46, 2-12.

Krojer, T., Garrido-Franco, M., Huber, R., Ehrmann, M. \& Clausen, T. (2002). Crystal structure of DegP (HtrA) reveals a new proteasechaperone machine. Nature 416, 455-459.

Lipinska, B., Zylicz, M. \& Georgopoulos, C. (1990). The HtrA (DegP) protein, essential for Escherichia coli survival at high temperatures, is an endopeptidase. J Bacteriol 172, 1791-1797.

Lu, B. \& McBride, B. C. (1994). Stress response of Porphyromonas gingivalis. Oral Microbiol Immunol 9, 166-173.

Lynch, M. C. \& Kuramitsu, H. K. (1999). Role of superoxide dismutase activity in the physiology of Porphyromonas gingivalis. Infect Immun 67, 3367-3375.

Lyon, W. R. \& Caparon, M. G. (2004). Role for serine protease HtrA (DegP) of Streptococcus pyogenes in the biogenesis of virulence factors SpeB and the hemolysin streptolysin S. Infect Immun 72, 1618-1625.

Maeda, H., Miyamoto, M., Hongyo, H., Nagai, A., Kurihara, H. \& Murayama, Y. (1994). Heat shock protein 60 (GroEL) from Porphyromonas gingivalis: molecular cloning and sequence analysis of its gene and purification of the recombinant protein. FEMS Microbiol Lett 119, 129-136.

Murakami, Y., Imai, M., Mukai, Y., Ichihara, S., Nakamura, H. \& Yoshimura, F. (2004a). Effects of various culture environments on expression of major outer membrane proteins from Porphyromonas gingivalis. FEMS Microbiol Lett 230, 159-165.

Murakami, Y., Masuda, T., Imai, M., Iwami, J., Nakamura, H., Noguchi, T. \& Yoshimura, F. (2004b). Analysis of major virulence factors in Porphyromonas gingivalis under various culture temperatures using specific antibodies. Microbiol Immunol 48, 561-569.

Mutunga, M., Graham, S., De Hormaeche, R. D., Musson, J. A., Robinson, J. H., Mastroeni, P., Khan, C. M. \& Hormaeche, C. E. (2004). Attenuated Salmonella typhimurium htrA mutants cause fatal infections in mice deficient in NADPH oxidase and destroy NADPH oxidase-deficient macrophage monolayers. Vaccine 22, 4124-4131.

Nakagawa, T., Saito, A., Hosaka, Y. \& Ishihara, K. (2003). Gingipains as candidate antigens for Porphyromonas gingivalis vaccine. Keio J Med 52, 158-162.
Nakayama, K. (2003). Molecular genetics of Porphyromonas gingivalis: gingipains and other virulence factors. Curr Protein Pept Sci 4, 389-395.

Noone, D., Howell, A. \& Devine, K. M. (2000). Expression of $y k d A$, encoding a Bacillus subtilis homologue of HtrA, is heat shock inducible and negatively autoregulated. J Bacteriol 182, 1592-1599.

Okuda, K. \& Ebihara, Y. (1998). Relationships between chronic oral infectious diseases and systemic diseases. Bull Tokyo Dent Coll 39, 165-174.

Olango, G. J., Roy, F., Sheets, S. M., Young, M. K. \& Fletcher, H. M. (2003). Gingipain RgpB is excreted as a proenzyme in the vimAdefective mutant Porphyromonas gingivalis FLL92. Infect Immun 71, 3740-3747.

Pallen, M. J. \& Wren, B. W. (1997). The HtrA family of serine proteases. Mol Microbiol 26, 209-221.

Percival, R. S., Marsh, P. D., Devine, D. A., Rangarajan, M., AduseOpoku, J., Shepherd, P. \& Curtis, M. A. (1999). Effect of temperature on growth, hemagglutination, and protease activity of Porphyromonas gingivalis. Infect Immun 67, 1917-1921.

Poquet, I., Saint, V., Seznec, E., Simoes, N., Bolotin, A. \& Gruss, A. (2000). HtrA is the unique surface housekeeping protease in Lactococcus lactis and is required for natural protein processing. Mol Microbiol 35, 1042-1051.

Potempa, J., Mikolajczyk-Pawlinska, J., Brassell, D., Nelson, D., Thogersen, I. B., Enghild, J. J. \& Travis, J. (1998). Comparative properties of two cysteine proteinases (gingipains R), the products of two related but individual genes of Porphyromonas gingivalis. J Biol Chem 273, 21648-21657.

Sculley, D. V. \& Langley-Evans, S. C. (2002). Salivary antioxidants and periodontal disease status. Proc Nutr Soc 61, 137-143.

Teng, Y. T., Taylor, G. W., Scannapieco, F., Kinane, D. F., Curtis, M., Beck, J. D. \& Kogon, S. (2002). Periodontal health and systemic disorders. J Can Dent Assoc 68, 188-192.

Travis, J., Banbula, A. \& Potempa, J. (2000). The role of bacterial and host proteinases in periodontal disease. Adv Exp Med Biol 477, 455-465.

van Ham, M. \& Hendriks, W. (2003). PDZ domains - glue and guide. Mol Biol Rep 30, 69-82.

Vanterpool, E., Roy, F. \& Fletcher, H. M. (2004). The vimE gene downstream of $\operatorname{vim} A$ is independently expressed and is involved in modulating proteolytic activity in Porphyromonas gingivalis W83. Infect Immun 72, 5555-5564.

Vanterpool, E., Roy, F., Zhan, W., Sheets, S. M., Sangberg, L. \& Fletcher, H. M. (2006). VimA is part of the maturation pathway for the major gingipains of Porphyromonas gingivalis W83. Microbiology 152, 3383-3389.

Wolff, L. F., Koller, N. J., Smith, Q. T., Mathur, A. \& Aeppli, D. (1997). Subgingival temperature: relation to gingival crevicular fluid enzymes, cytokines, and subgingival plaque micro-organisms. J Clin Periodontol 24, 900-906.

Wonderling, L. D., Wilkinson, B. J. \& Bayles, D. O. (2004). The htrA ( $\operatorname{deg} P$ ) gene of Listeria monocytogenes 10403S is essential for optimal growth under stress conditions. Appl Environ Microbiol 70, 1935-1943.

Yamazaki, K., Ueki-Maruayama, K., Honda, T., Nakajima, T. \& Seymour, G. J. (2004). Effect of periodontal treatment on the serum antibody levels to heat shock proteins. Clin Exp Immunol 135, 478-482.

Yoshida, A., Nakano, Y., Yamashita, Y., Oho, T., Shibata, Y., Ohishi, M. \& Koga, T. (1999). A novel dnaK operon from Porphyromonas gingivalis. FEBS Lett 446, 287-291. 